\title{
IMPACT OF ORGANIZED PHYSICAL EXERCISE ON QUALITY OF LIFE AND FATIGUE IN CHILDREN AND ADOLESCENTS WITH CANCER
}

\author{
Filippo Spreafico ${ }^{1}$, Francesco Barretta ${ }^{2}$, Michele Murelli ${ }^{3}$, Marco Chisari ${ }^{2}$, Giovanna \\ Gattuso $^{2}$, Monica Terenziani ${ }^{2}$, Andrea Ferrari ${ }^{4}$, Laura Veneroni ${ }^{5}$, Cristina Meazza ${ }^{6}$, and \\ Maura Massimino ${ }^{7}$ \\ ${ }^{1}$ Fondazione IRCCS Istituto Nazionale Tumori \\ ${ }^{2}$ Fondazione IRCCS Istituto Nazionale dei Tumori \\ ${ }^{3}$ Physiotherapy Center Murelli Vergnaghi s.r.l. \\ ${ }^{4}$ Istituto Nazionale Tumori, Milano \\ ${ }^{5}$ Pediatric Oncology Unit, Fondazione IRCCS Istituto Nazionale Tumori, Milano \\ ${ }^{6}$ Istituto Nazionale Tumori, Milano \\ ${ }^{7}$ Istituto Nazionale Tumori
}

July 30, 2020

\begin{abstract}
Background: Children and adolescents with cancer are at risk of a poor health-related quality of life. Exercise interventions to enhance movement may be a valid strategy for managing some symptoms, including fatigue. Methods: Forty-four consecutive patients (20 females; aged 5-21 years old, median 15.5 years), without any contraindications significantly limiting their movements were invited to join an in-hospital 6-week supervised exercise program, and asked afterwards to complete the PedsQL-4.0 quality of life Generic Core Scales and the PedsQL Multidimensional Fatigue Scale. The program consisted of personalized workout sessions of aerobic, resistance and flexibility exercises. The results obtained on the scales were compared between patients who engaged in the exercise program (GYM group, n=21) and those who did not (No-GYM, n=23), with the aim of examining the different dimensions of health-related quality of life (physical, emotional, cognitive, social) and fatigue (general, sleep/rest, cognitive) comparing the two groups. Results: For 43 of 44 patients, being diagnosed with cancer initially prompted a drop-out from previous physical exercise or sports routines despite no contraindications to their continuation. After 6 weeks, the scores for patients in the GYM group showed a statistically significant better perceived emotional functioning, and a possible indication of improved social functioning compared with the No-GYM group. Conclusion: These findings suggest that to exercise improves the satisfaction of children and adolescents with cancer with their physical, mental and social functioning. It is worth further investigating the value of systematically including exercise workouts in their routine cancer practices.
\end{abstract}

Main text word count: 3494

Number of tables: 4

Number of figures: 1

KEYWORDS : patient-reported outcomes, health-related quality of life, exercise, physical activity, pediatric cancer, cancer-related fatigue

SHORT RUNNING TITLE : PHYSICAL EXERCISE IN PEDIATRIC ONCOLOGY 


\title{
ABBREVIATIONS
}

\begin{tabular}{ll}
\hline Health-related quality of life & HRQoL \\
\hline Interquartile Range & IQR \\
Quality of Life & QoL \\
\hline
\end{tabular}

\begin{abstract}
Background : Children and adolescents with cancer are at risk of a poor health-related quality of life. Exercise interventions to enhance movement may be a valid strategy for managing some symptoms, including fatigue.
\end{abstract}

Methods: Forty-four consecutive patients (20 females; aged 5-21 years old, median 15.5 years), without any contraindications significantly limiting their movements were invited to join an in-hospital 6-week supervised exercise program, and asked afterwards to complete the PedsQL-4.0 quality of life Generic Core Scales and the PedsQL Multidimensional Fatigue Scale. The program consisted of personalized workout sessions of aerobic, resistance and flexibility exercises. The results obtained on the scales were compared between patients who engaged in the exercise program (GYM group, $n=21$ ) and those who did not (No-GYM, $n=23$ ), with the aim of examining the different dimensions of health-related quality of life (physical, emotional, cognitive, social) and fatigue (general, sleep/rest, cognitive) comparing the two groups.

Results: For 43 of 44 patients, being diagnosed with cancer initially prompted a drop-out from previous physical exercise or sports routines despite no contraindications to their continuation. After 6 weeks, the scores for patients in the GYM group showed a statistically significant better perceived emotional functioning, and a possible indication of improved social functioning compared with the No-GYM group.

Conclusion: These findings suggest that to exercise improves the satisfaction of children and adolescents with cancer with their physical, mental and social functioning. It is worth further investigating the value of systematically including exercise workouts in their routine cancer practices.

\section{INTRODUCTION}

Children and adolescents with cancer are generally treated with chemotherapy, sometimes combined with radiotherapy and/or surgery. Their disease and its treatments can cause a variety of early and late morbidities, including fatigue, neuropathy, sleep disruption, chronic pain, depression, anxiety, cognitive impairment, and a worse quality of life (QoL). ${ }^{1-3}$ Overall, the physical functioning of children and adolescents with cancer declines during their treatment, and this can interfere with their QoL, ${ }^{4-6}$ as well as increasing the risk of certain chronic diseases. $^{2}$

There has been growing interest in studying the value of exercise in oncology, albeit more in adults than in pediatric patients so far. ${ }^{7-11}$ Regular exercise can help patients receiving therapy and cancer survivors to avoid becoming trapped in a self-perpetuating cycle of deteriorating physical functioning that can exacerbate the negative consequences of fatigue and a sedentary lifestyle..$^{5,11-13}$

Health-related QoL (HRQoL) is a construct that describes a person's perception of their own physical, mental and social wellbeing. ${ }^{14-17}$ Positive associations have been reported between HRQoL and fitness levels in childhood cancer survivors. ${ }^{18,19}$ Cancer-related fatigue is a common and distressing condition that significantly lowers the HRQoL of adults and children with cancer, ${ }^{20,21}$ and higher levels of physical activity have been associated with lower levels of cancer-related fatigue. ${ }^{22}$ It is worth also to consider that in children the routine use of systemic pharmacological approaches to manage fatigue is not generally recommended. ${ }^{23}$

Physical vitality is an important marker of any teenager's physical and psychosocial health, be they healthy or ill, and higher levels of physical activity have been found to correlate with better cognitive outcomes. ${ }^{24-27}$ Despite a wider acknowledgement of the value of movement for patients with cancer, the prescription of exercise in oncology has remained somewhat basic, particularly in the case of children. With the aim of 
improving the opportunities for children and adolescents with cancer to exercise, we have implemented an in-hospital training program at the pediatric department of our institution. In an effort to identify optimal approaches (or barriers) to organized exercise programs in clinical cancer practice, this study aimed: (i) to identify any improvement in physical, psychosocial and emotional functioning in children and adolescents with various solid cancers after the introduction of supervised controlled exercising programs; (ii) to compare HRQoL scores between patients who attended the workouts and those who did not; and (iii) to investigate barriers to physical activity and participation in sport for young people diagnosed with cancer.

\section{METHODS}

\section{Participants and study design}

Between April and August 2018, consecutive patients were invited to attend professionally-supervised sessions of physical exercise in the hospital $\mathrm{gym}^{28}$ as part of this study. The exercise sessions each lasted one hour, and included combinations of cardiovascular training, strength and endurance exercises, relaxation, and muscle stretching. They were prescribed individually for each patient, offered three times a week, for a total duration of 6 weeks.

Inclusion criteria were: age [?]5 years and less than 21 years; a diagnosis of solid cancer; the ability to communicate in Italian. The only exclusion criterion was the presence of limitations (disability and/o severe morbidities) preventing any level of physical activity. In principle, severe disabilities (e.g. lower extremity amputation, or palsy) were not regarded as a contraindication if patients were able to exercise with adapted personalized workouts involving the less compromised parts of their body.

Based on their own decision to join the exercise program or not, the patients involved in the study formed two groups (GYM and No-GYM, respectively) and were compared in descriptive terms. Consent to participation in the study was obtained from all patients and/or their guardians. The institutional Ethics Committee approved the study (INT 109/18) and the data protection methods.

\section{Data collection}

Information on patients' physical activity levels and habits prior to being diagnosed with cancer, their personal attitudes to participation in sports, and their knowledge and expectations of the institutional physical exercise program was obtained using an ad hoc questionnaire. Data on patients' diagnoses, demographics and treatments were retrieved from their medical records.

The 23-item multidimensional PedsQL-4.0 Generic Core Scales ${ }^{14,17}$ (Italian edition) comprise 4 scales measuring: physical functioning, emotional functioning, social functioning, and school functioning. Answers are scored on a 5-point rating scale ranging from 'never' to 'almost always'. The total score is calculated as the average of the scores for each item. Total scores were converted into a score ranging from 0 to 100 , with higher scores indicating a better QoL for functioning.

The 18-item PedsQL Multidimensional Fatigue Scale ${ }^{15,16,29}$ (Italian edition) includes three subscales (with six items each) for general fatigue, sleep/rest fatigue, and cognitive fatigue. Respondents are asked to rate how much of a problem each item has been during the past month on a 5-point Likert scale $(0=$ never, $1=$ almost never, $2=$ sometimes, $3=$ often, $4=$ almost always).

Child self-reports are age-specific, for age 5-7 years (young child), 8-12 years (child), and 13-18 years (adolescent). Older patients were administered the PedsQL Young Adult Report (ages 18-25 years).

The team's psychologists, exercise professionals, or oncologists were available for patients and their parents to provide assistance regarding the self-administered questionnaires. The questionnaires were administered to the GYM group after participants had completed the 6-week training program, and to the No-GYM group at the baseline.

\section{Statistical methods}


The results obtained from the questionnaires were analyzed using classical descriptive statistics for the study population as a whole, and by study group to compare patients who attended the gym (GYM group) with those who did not (No-GYM group). The scores were calculated with the PedsQL 4.0 scoring algorithm, and the medians and interquartile ranges (IQR) were reported for each item and each dimension, and for total scores (if any), for each PedsQL questionnaire.

Differences between the two groups in terms of patients' characteristics, and their scores in the questionnaires (by single dimension and total scores) were assessed with Fisher's exact test or the Wilcoxon test. Possible correlations between total scores (for physical health, psychosocial health, and fatigue) were computed using Pearson's correlation index.

The statistical analyses were performed with SAS (SAS Institute, Cary, NC) and R software (R Foundation for Statistical Computing, Vienna, Austria).

\section{RESULTS}

\section{General results}

Altogether, the study participants included 44 patients who completed the self-administered HRQoL and general questionnaires involved in the study. The median age of the whole sample was 15.5 years, and 20/44 patients were female. Twenty-one patients (48\%) attended the proposed exercise sessions, and thus formed the GYM group, while $23(52 \%)$ refused the proposed exercise program and formed the No-GYM group. The two groups were statistically different as regards type of cancer (P 0.034): 6 of 8 patients with brain tumors refused to attend the gym; and all patients with neuroblastoma invited joined the GYM group. Table 1 shows patients' characteristics and their answers in the general questionnaire, overall and by group.

\section{General questionnaire}

Overall, 27 patients $(61 \%)$ reported having exercised regularly before their tumor was diagnosed, with no statistically significant difference between the two groups (14/21 [67\%] in the GYM group, and 13/23 [56\%] in the No-GYM). Leaving aside three patients who reportedly did not exercise even before they were diagnosed with cancer, $32 / 41$ patients (78\%) reported completely dropping out of any physical activity when their disease was diagnosed, 8/41(20\%) significantly reduced their level of exercise, and only one continued to exercise as before. The main reasons for limiting their exercise or sports or dropping out were: fatigue (26/40); lack of time (11/40, partly due to frequent hospital visits); physical disabilities (amputation, in 1; problems leading to the inability to walk in 5); and the presence of a central venous device (2). Seventeen patients $(43 \%)$ were discouraged from continued exercising because their tumor was regarded in itself as a contraindication (by general practitioners in 9 cases, by parents in 3 cases, by the patients themselves in 4 cases, and by the coach of their usual sports activity in 1 case).

Seventeen patients had already attended the hospital gym before this study was launched (and 12 of them reported not exercising regularly or engaging in any sports before their tumor was diagnosed), and were asked some additional questions referring to the period before the study began. Their reported motives for attending the gym were: to keep fit $(8 / 17)$; to combat treatment-related side effects $(3 / 17)$; to take their mind off their situation $(12 / 17)$; to have an opportunity to socialize with their peers $(2 / 17)$; to heal their relationship with their bodies (6). After starting to attend the hospital gym, they reported experiencing the following benefits: they felt physically fitter (8); they were better able to manage their anger (11), sadness (12), irritability (11), or anxiety (9); it helped to control side effects of treatment (nausea, vomiting), and improved their relationships with peers (5). Eleven of the 17 patients would have advised other patients to use the hospital gym. All 17 patients said they would not be worried about resuming their physical activities after completing chemotherapy, and 15 (88\%) said they would come back to the hospital gym even after completing their cancer treatments, during their follow-up as outpatients (to get fitter before going to other gyms in 5 cases, to keep in touch with other patients in 5 , and because the in-hospital gym was seen as a more 'protected' area in 5).

\section{Results of the PedsQL-4.0 Generic Core and PedsQL Fatigue Multidimensional scales}


Table 2 shows the scores obtained in the questionnaires, by single dimension and in total, in the two study groups, and Table 3 shows the scores for single items within each dimension.

The median scores (and IQRs) for perceived emotional functioning were 85.0 (62.5-87.5) and 60.0 (45.0$75.0)$ in the GYM group and No-GYM groups $(\mathrm{p}=0.018)$, respectively. This is the only dimension in which statistically significant different results emerged between the two groups. Specific items associated with this difference were: fear, sadness, sleeping difficulties, and uncertainty about the future, with median values of 100 (IQR: 75-100) versus 75 (IQR: 50-100), 75 (IQR: 50-100) versus 50 (IQR: 50-75), 100 (IQR: 50-100) versus 75 (IQR: 50-100), and 100 (IQR: 50-100) versus 50 (IQR: 25-75), respectively, for the GYM group versus the No-GYM group.

For social functioning, the GYM group returned slightly higher scores than the No-GYM group for the items concerning getting along with peers (median 100 [IQR: 75-100] versus 75 [IQR: 75-100]), and making friends (median 100 [IQR: 100-100] versus 75 [IQR: 75-100]). For the item about being able to do what peers can do, on the other hand, the GYM group had lower scores than the No-GYM group (median 62.5 [IQR: 50-87.5] versus 75 [IQR: 50-100]).

The psychosocial functioning of the GYM group was also reported to be slightly better than that of the No-GYM group (median 76.7 [IQR: 67.5-83.3] versus 71.7 [IQR: 56.7-76.7]).

The median HRQoL scores obtained with the PedsQL Fatigue scale were lower for the GYM group than in the No-GYM group, except for the "general fatigue" dimension, which was a median 66.7 [IQR: 58.3-79.2] for the GYM group as opposed to 62.5 [IQR: 50-83.3] for the No-GYM, group. On analyzing the different items, the median scores were found higher in the GYM group than in the No-GYM group for perceived sleep quality (median 100 [IQR: 50-100] versus 87.5 [IQR: 50-100]) and for fatigue in activities of daily living (median 100 [IQR: 75-100] versus 87.5 [IQR: 75-10]).

The correlations between total scores (for physical health, psychosocial health, and fatigue) were all weakly positive, ranging between 0.51 (for physical health and fatigue) and 0.68 (for psychosocial health and fatigue) (according to Pearson's correlation index; Table 4 and Figure 1).

\section{DISCUSSION}

The importance of physical activity for individuals during and after cancer care has been increasingly recognized. It has favorable effects on various levels of functioning, encompassing aspects of QoL, mood symptoms, fitness level, muscle strength, body composition, and active attainment of social roles. ${ }^{5,7,8,12,24,30,31}$ A possible relationship between the level of fitness and the risk of both mortality ${ }^{4}$ and tumor recurrence ${ }^{11,32}$ has also emerged, and is worth exploring.

We know that adolescent and young adults with cancer are less active than their healthy peers (especially when in hospital), and that many of them experience important morbidities while receiving treatment. . $^{3,28,29}$

Fatigue has multiple dimensions that may involve physical, psychological, social and cognitive aspects of the person affected. Consequences of fatigue causes the inability to engage in everyday life activities and social roles (including educational and work opportunities), mood swings, sleep disturbances, impaired relations, lower academic achievement, and impaired QoL. ${ }^{21,22}$ Among pediatric patients receiving cancer treatments, fatigue is more prevalent in adolescents, and might be particularly important in this age group. ${ }^{20,33}$

When interviewed at the baseline, $97.5 \%$ of our patients who were active before being diagnosed with cancer reported a substantial decline in their exercising and sports activities compared with their previous levels: the main reasons mentioned for abandoning included fatigue, lack of time, and physical disabilities. It is noteworthy that $42 \%$ of these patients suspended their physical activities because they had been told, wrongly, that they were contraindicated for cancer patients. This is a common misconception even among general practitioners.

The purpose of our study was to explore the potential effects of an organized exercise program for children and adolescents at a comprehensive cancer center (where a structured exercise program is available ${ }^{28}$ ) on 
patients' self-reported HRQoL and fatigue.

Our findings indicate that patients who attended the exercise sessions (our GYM group) perceived a better emotional functioning than those who did not, obtaining significantly better scores especially forfear , sadness, sleeping difficulties, anduncertainty about the future. We know that the benefits of exercising and practicing sports go far beyond physical function endpoints alone, but there are still gaps in our understanding of how they benefit the emotional and social spheres, and resilience as well. ${ }^{13,27}$ A key finding in a study on a large cohort of childhood cancer survivors was that vigorous exercise was associated with a lower risk of depression, somatization, and cognitive impairment. ${ }^{31}$ Training for a sports competition might symbolize patients' return to setting themselves healthy and challenging plans for their future. Endorphins, endocannabinoids, monoamines, and neurotrophins have all been implicated in the euphoric response to endurance running, ${ }^{34}$ and may reinforce the biological rationale behind some of our findings. ${ }^{35}$ Other investigations have suggested that cardiorespiratory fitness may directly affect brain function, or have attributed the psychological benefits of exercise to improvements in sleep duration or quality. ${ }^{36}$

Looking at the positive effects of exercising on emotionalhealth, martial arts classes that were recently added to our in-hospital exercise programs ${ }^{13}$ have proved an interesting way to empower our patients emotionally too, improving their breathing and relaxation skills. During these classes, most of the work aims to help young patients to cope with feelings of pain, anger, anxiety, fear, loss of control, and diminished self-esteem. Interestingly, a panel of experts made a strong recommendation for the use of relaxation techniques or mindfulness, or both, to manage cancer-related fatigue, based on consistent findings concerning the benefits of these practices across different types of adult patients and intervention. ${ }^{23}$

A better emotional health and self-esteem can also relate to a better social functioning. Patients in our GYM group had better scores for some items referring to social functioning (e.g.getting along with peers, making friends ). For adolescents especially, the benefits of engaging in sports might include regaining a sense of having a lively, properly-functioning body, enhancing relationship with peers and a sense of independence. These findings are in line with research demonstrating positive relationships between physical activity and cognitive outcomes. ${ }^{24-26,37}$

For the youngest children, integrating physical education in hospital routines may also be important not only to promote physiological motor development, ${ }^{38,39}$ but also to preserve or restore a normality that cancer patients often lack, such as a chance to play with friends like during gym classes at school. It is important to identify approaches to patients' safe practice of physical activities, taking into account their age-specific preferences. We know that encouraging physical activity in small children is challenging, but we found that children as young as 5 years old could take an active part in the workout sessions considered in the present study.

On the other hand, our GYM group had worse scores than the No-GYM group for the item that concerned being able to do what peers can do. In principle, everybody can find a suitable form of exercise or sport to practice, though a delicate balance has to be struck between individuals' wishes and their capabilities (which may have been irreparably affected by their cancer). This may demand adapting certain sports to patients with disabilities, or providing parallel psychological support in some circumstances. Asking patients to exercise may exacerbate their frustrations if the demands placed on them exceed their expected capabilities, especially if prescribed levels of physical fitness are aligned with those enjoyed by a patient before they were diagnosed with cancer.

Our two groups (GYM and No-GYM) differed in terms of cancer types. Indeed patients with brain tumors - who are known to suffer from neuromotor impairments ${ }^{40}$ - seemed more reluctant to attend the gym (6 out of 8 such patients refused). Professionally-supervised programs need to be developed to accommodate underlying organ system impairments, so that alternative, customized workouts can be used to exercise the less impaired parts of a patient's body. It is worth noting, for instance, how any lower limb impairment preventing a patient from walking tended to be misconceived as a major reason for not exercising at all because such patients were unable to walk, run, jump, or kick a ball (but they could work out effectively 
with their upper limbs and trunk).

The strengths of our study include our having examined several dimensions of HRQoL and fatigue, and the correlations between the three dimensions of physical health, psychological functioning and fatigue as a whole. Individuals who have a more positive perception of their neurocognitive functioning may also experience greater self-efficacy, and this would make it easier for them to engage in exercising. The direct correlation identified in our sample between a better perceived physical functioning and a greater perception of fatigue probably stems from the fact that patients motivated to exercise and try to overcome their limits also had a more acute awareness of these limits and their sense of fatigue.

Our findings indicate that children attending the gym scored worse on fatigue, except for general fatigue - Analyzing the single items in the questionnaire showed that the median scores were better in the GYM group for perceived quality of sleep and daily fatigue. The effect of exercise on the severity of fatigue may differ, depending on the type of activity involved (aerobic, neuromotor, or combined exercises might be more effective in containing fatigue than resistance exercises ${ }^{23}$ ), and the intensity of the exercise. Given the very heterogeneous nature of cancer populations, recent publications have called for a more precise manipulation of training variables - such as volume, intensity, and frequency - to truly optimize clinically-relevant patientreported outcomes. ${ }^{10} \mathrm{We}$ did not homogeneously prescribe or record the training parameters adopted in our sample, however, so we are unable to say which type of exercise might be best exercise for the study outcomes, or why scores for fatigue were worse for the GYM group.

There are other limitations in our study to acknowledge. Patients were assigned to one of the two groups being compared not randomly, but based on their volunteering for the exercise program. The patients' reasons for attending the training sessions may have been positively or negatively influenced by their ability (or fear of inability) to cope with the exercises, or by their attitude to sports before becoming ill (although a similar proportion of patients in the GYM and No-GYM groups had not exercised regularly before being diagnosed with cancer). Our patients also formed a very heterogeneous group, with ages ranging from 5 to 21 years. They had different types of solid tumor, and received different treatments, sometimes involving surgery and radiotherapy as well as chemotherapy (which was administered to all participants in the study). This means that any differences between the GYM and No-GYM groups may not be attributable to our exercise program alone, but also to other factors not assessed (e.g., changes in health status, medication, or other cancer treatments).

A further limitation includes the fact that no longitudinal data were available (to enable any within-subject comparison between before and after the 6-week exercise program in the self-reported QoL outcomes for patients in the GYM group).

We hope our findings will attract attention to the feasibility and possible benefits of promoting more systematic opportunities to move and exercise for young cancer patients, even during their active treatment, to improve their self-reported physical and psychosocial symptoms and HRQoL, and especially their emotional functioning. It is likely that keeping physically active helps. The results of our baseline survey also suggest that many young patients with cancer, their parents, and their doctors feel that a diagnosis of cancer makes it unsafe or unfeasible to exercise and practice sports, but this is a modifiable barrier.

CONFLICTS OF INTEREST STATEMENT: The authors have no conflict of interest to disclose.

AVAILABILITY OF DATA AND MATERIAL The datasets generated and/or analyzed as part of the present study are available from the corresponding author on reasonable request.

ACKNOWLEDGMENTS: We thank the Associazione Bianca Garavaglia Onlus for their constant financial support. We are also grateful to Gloria and Pasquale, sport professionals, who assist our patients with tailored workouts and constant commitment. The research was partially supported by a private grant from the Associazione Bianca Garavaglia onlus (salaries for sports professionals).

\section{REFERENCES}


1. Ahlberg K, Ekman T, Gaston-johansson F, et al. Assessment and Management of Cancer-related Fatigue in adults. Lancet. 2003;362:640-50.

2. Bhakta N, Liu Q, Ness KK, et al. The Cumulative Burden of Surviving Childhood Cancer: an Initial Report from the St. Jude Lifetime Cohort Study. Lancet. 2017; 390(10112): 2569-2582.

3. Hoffman MC, Mulrooney DA, Steinberger J, et al. Deficits in Physical Function Among Young Childhood Cancer Survivors. J Clin Oncol. 2020;31:2799-805.

4. Lear SA, Hu W, Rangarajan S, et al. The Effect of Physical Activity on Mortality and Cardiovascular Disease in 130000 People from 17 High-income, Middle-income, and Low-income Countries: the PURE Study. Lancet. 2017;390:2643-2654.

5. Howell CR, Wilson CL, Ehrhardt MJ, et al. Clinical Impact of Sedentary Behaviors in Adult Survivors of Acute Lymphoblastic Leukemia: a Report from the St. Jude Lifetime Cohort Study. Cancer. 2018. 124(5): 1036-1043.

6. Wilson CL, Stratton K, Leisenring WL, et al. Decline in Physical Activity Level in the Childhood Cancer Survivor Study Cohort. Cancer Epidemiol Biomarkers Prev. 2014;1619-28.

7. Winter C, Müller C, Hoffmann C, et al. Physical Activity and Childhood Cancer. Pediatr. Blood Cancer. 2010; 54:501-510.

8. Wolin KY, Ruiz JR, Tuchman H, et al. Exercise in Adult and Pediatric Hematological Cancer Survivors: An Intervention Review. Leukemia. 2010;24:1113-20.

9. Mina DS, Sabiston CM, Au D, et al. Connecting People with Cancer to Physical Activity and Exercise Programs: A Pathway to Create Accessibility and Engagement. Curr Oncol. 2018;25:149-62.

10. Mina DS, Langelier D, Adams SC, et al. Exercise as Part of Routine Cancer Care. Lancet Oncol. 2018; 19:e433-e436.

11. McTiernan A, Friedenreich CM, Katzmarzyk PT, et al. Physical Activity in Cancer Prevention and Survival: a Systematic Review. Med Sci Sports Exerc. 2019; 51(6): 1252-1261.

12. Rustler V, Hagerty M, Daeggelmann J, et al. Exercise Interventions for Patients with Pediatric Cancer During Inpatient Acute Care: A Systematic Review of Literature. Pediatr. Blood Cancer. 2017;64(11).

13. Spreafico F, Murelli M, Timmons BW, et al. Sport Activities and Exercise as Part of Routine Cancer Care in Children and Adolescents. Pediatr Blood Cancer. 2019;66(8):e27826.

14. Varni JW, Burwinkle TM, Katz ER, et al. The PedsQL ${ }^{\mathrm{TM}}$ in Pediatric Cancer: Reliability and Validity of the Pediatric Quality of Life Inventory ${ }^{\mathrm{TM}}$ Generic Core Scales, Multidimensional Fatigue Scale, and Cancer Module. Cancer. 2002;Apr 1 ;94(7):2090-106.

15. Varni JW, Burwinkle TM, Szer IS. The PedsQL ${ }^{\mathrm{TM}}$ Multidimensional Fatigue Scale in Pediatric Rheumatology: Reliability and Validity. J Rheumatol. 2004 Dec;31(12):2494-500.

16. Varni JW, Limbers CA. The PedsQL ${ }^{\mathrm{TM}}$ Multidimensional Fatigue Scale in Young Adults: Feasibility, Reliability and Validity in a University Student Population. Qual Life Res. 2008 Feb;17(1):105-14.

17. Varni JW, Seid M, Rode CA. The PedsQL ${ }^{\mathrm{TM}}$ : Measurement Model for the Pediatric Quality of Life Inventory. Med Care. 1999;37(2):126-139.

18. Badr H, Chandra J, Paxton RJ, et al. Health-related Quality of Life, Lifestyle Behaviors, and Intervention Preferences of Survivors of Childhood Cancer. J Cancer Surviv. 2013;7:523-34.

19. Medysky ME, Temesi J, Culos-Reed SN, et al. Exercise, Sleep and Cancer-related Fatigue: Are They Related? Neurophysiol Clin Neurophysiol. 2017;47(2):111-122. 
20. van Deuren S, Boonstra A, van Dulmen-den Broeder E, et al. Severe Fatigue After Treatment for Childhood Cancer. Cochrane database Syst. Rev. 2020; 3;3:CD012681.

21. Bower JE. Cancer-related Fatigue: Mechanisms, Risk Factors, and Treatments. Nat Rev Clin Oncol. 2014; 11(10): 597-609.

22. Van Dijk-Lokkart EM Van, Veening MA, Merks JH, et al. Longitudinal Development of Cancer-related Fatigue and Physical Activity in Childhood Cancer Patients. Pediatr Blood Cancer. 2019;66(12):e27949.

23. Robinson PD, Oberoi S, Tomlinson D, et al. Management of Fatigue in Children and Adolescents with Cancer and in Paediatric Recipients of Haemopoietic Stem-cell Transplants: a Clinical Practice Guideline. Lancet Child Adolesc. Heal. 2018 May;2(5):371-378.

24. Barlow-Krelina E, Chen Y, Yasui Y, et al. Consistent Physical Activity and Future Neurocognitive Problems in Adult Survivors of Childhood Cancers : A Report From the Childhood Cancer Survivor Study. J Clin Oncol. 2020; 38(18):2041-2052.

25. Cox EP, O'Dwyer N, Cook R, et al. Relationship between Physical Activity and Cognitive Function in Apparently Healthy Young to Middle-age Adults: a Systematic Review. J Sci Med Sport. 2016; 19(8):616628.

26. Phillips NS, Howell CR, Lanctot JQ, et al. Physical Fitness and Neurocognitive Outcomes in Adult Survivors of Childhood Acute Lymphoblastic Leukemia: A Report From the St . Jude Lifetime Cohort. Cancer. 2020; 126:640-648.

27. Rodriguez M, Cristina A, Sanchez C, et al. Role of Physical Activity and Sedentary Behavior in the Mental Health of Preschoolers, Children and Adolescents : A Systematic Review and Meta - Analysis. Sport Med. 2019;49(9):1383-410.

28. Spreafico F, Murelli M, Ferrari A, et al. Should We Encourage Exercise and Sports in Children and Adolescents with Cancer? Pediatr. Blood Cancer. 2014;61:2125.

29. Daniel LC, Brumley LD, Schwartz LA. Fatigue in Adolescents with Cancer Compared to Healthy Adolescents. Pediatr Blood Cancer. 2013;60(11):1902-7.

30. Scott JM, Zabor EC, Schwitzer E, et al. Efficacy of Exercise Therapy on Cardiorespiratory Fitness in Patients with Cancer: A Systematic Review and Meta-analysis. J Clin Oncol. 2018;36:2297-2305.

31. Tonorezos ES, Ford JS, Wang L, et al. Impact of Exercise on Psychological Burden in Adult Survivors of Childhood Cancer : A Report From the Childhood Cancer Survivor Study. 2019;125(17):3059-67.

32. Cormie P, Zopf EM, Zhang X, et al. The Impact of Exercise on Cancer Mortality, Recurrence, and Treatment-related Adverse Effects. Epidemiol Rev. 2017;39:71-92.

33. Nowe E, Stobel-Richter Y, Sender A, et al. Cancer-related Fatigue in Adolescents and Young Adults : A Systematic Review of the Literature. Crit Rev Oncol Hematol. 2017;118:63-69.

34. Hicks SD, Jacob P, Perez O, et al. The Transcriptional Signature of a Runner's High. Med Sci Sports Exerc. 2019;51(5):970-978.

35. Fairman CM, Zourdos MC, Helms ER, et al. A Scientific Rationale to Improve Resistance Training Prescription in Exercise Oncology. Sports Med. 2017; 47(8):1457-1465.

36. Heijnen S, Hommel B, Kibele A, et al. Neuromodulation of Aerobic Exercise -A Review. Front Psychol. $2015 ; 6: 1890$.

37. Riggs L, Piscione J, Laughlin S, et al. Exercise Training for Neural Recovery in a Restricted Sample of Pediatric Brain Tumor Survivors : a Controlled Clinical Trial with Crossover of Training Versus No Training. Neuro-oncol. 2017;19(3):440-450. 
38. Hotting K, Roder B. Beneficial Effects of Physical Exercise on Neuroplasticity and Cognition. Neurosci Biobehav Rev. 2013;37:2243-57.

39. Singh A, Uijtdewilligen L, Twisk JWR, et al. Physical Activity and Performance at School: A Systematic Review of the Literature Including a Methodological Quality Assessment. Arch Pediatr Adolesc Med. 2012; 166(1):49-55.

40. Piscione PJ, Bouffet E, Mabbott DJ, et al. Physical Functioning in Pediatric Survivors of Childhood Posterior Fossa Brain Tumors. Neuro-Oncology. 2014;16(1):147-155.

\section{FIGURE LEGENDS}

Figure 1. Scatter plots and spline curves of the correlation between the global assessment scores on the PedsQL-4.0 psychological (qolPSYCm) and physical (qolPHYSm) functioning scales, and the PedsQL-3.0Fatigue (FATm) scale for the cohort as a whole.

\section{Hosted file}

Table1.docx available at https://authorea.com/users/347483/articles/473153-impact-oforganized-physical-exercise-on-quality-of-life-and-fatigue-in-children-and-adolescentswith-cancer

\section{Hosted file}

Table2.docx available at https://authorea.com/users/347483/articles/473153-impact-oforganized-physical-exercise-on-quality-of-life-and-fatigue-in-children-and-adolescentswith-cancer

\section{Hosted file}

Table3.docx available at https://authorea.com/users/347483/articles/473153-impact-oforganized-physical-exercise-on-quality-of-life-and-fatigue-in-children-and-adolescentswith-cancer

\section{Hosted file}

Table4.docx available at https://authorea.com/users/347483/articles/473153-impact-oforganized-physical-exercise-on-quality-of-life-and-fatigue-in-children-and-adolescentswith-cancer
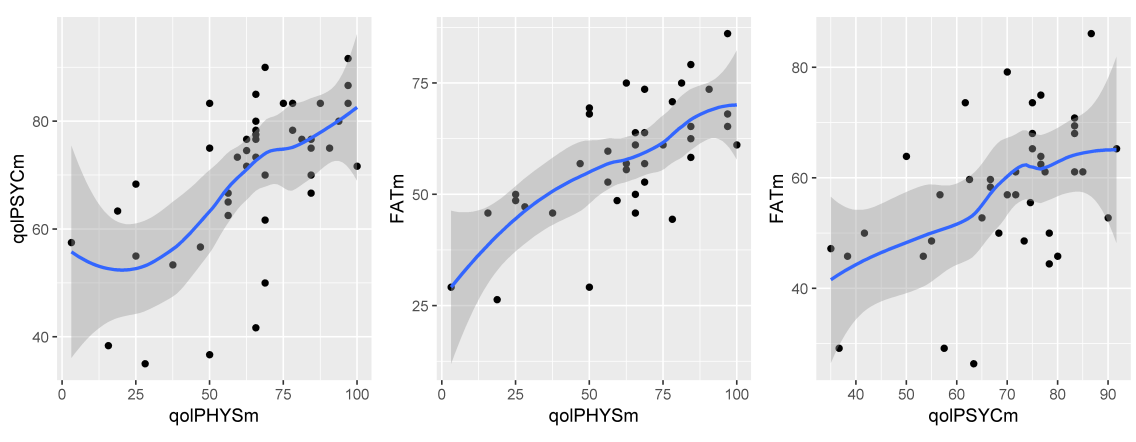